\title{
Microwave Dielectric Property Based Classification of Renal Calculi: Application of a kNN Algorithm
}

\author{
Banu Saçlı ${ }^{\mathrm{a}}$, Cemanur Aydınalp ${ }^{\mathrm{a}}$, Gökhan Cansız ${ }^{\mathrm{a}}$, Sulayman Joof ${ }^{\mathrm{a}}$, Tuba \\ Yilmaz $^{\mathrm{a}}$, Mehmet Çayören ${ }^{\mathrm{a}}$, Bülent Önal ${ }^{\mathrm{b}}$, and Ibrahim Akduman ${ }^{\mathrm{a}}$ \\ ${ }^{a}$ Department of Electronics and Communication Engineering \\ Istanbul Technical University, Istanbul, Turkey \\ ${ }^{b}$ Department of Urology, Cerrahpasa Medical School, \\ Istanbul University - Cerrahpasa, Istanbul, Turkey
}

\begin{abstract}
The proper management of renal lithiasis presents a challenge, with the recurrence rate of the disease being as high as $46 \%$. To prevent recurrence, the first step is the accurate categorization of the discarded renal calculi. Currently, the discarded renal calculi type is determined with the X-ray powder diffraction method which requires a cumbersome sample preparation. This work presents a new approach that can enable fast and accurate classification of discarded renal calculi with minimal sample preparation requirements. To do so, first, the measurements of the dielectric properties of naturally formed renal calculi are collected with the open-ended contact probe technique between $500 \mathrm{MHz}$ to 6 $\mathrm{GHz}$ with $100 \mathrm{MHz}$ intervals. Cole-Cole parameters are fitted to the measured dielectric properties with the generalized Newton-Raphson method. The renal calculi types are classified based on their Cole-Cole parameters as calcium oxalate, cystine, or struvite. The classification is performed using nearest neighbors $(\mathrm{kNN})$ machine learning algorithm with the 10 nearest neighbors, where accuracy as high as $98.17 \%$ is achieved.
\end{abstract}

Keywords: Dielectric properties of renal calculi, kidney stone, open-ended coaxial probe, Cole-Cole parameters, classification of kidney stones, machine learning, k-nearest neighbors

\section{Introduction}

Renal lithiasis, defined as the biomineralization of the urinary system, affects $14 \%$ of the population globally 1, 2. The disease has to be well managed to prevent recurrence, which has been reported to be as high as $46 \%$ in five-year 5 follow-up patient studies [3]. Prevention of disease recurrence is possible via proper medication and dietary restrictions. However, to designate the appropriate prescription, the renal calculi types should be determined by analyzing the discarded material. There are four major renal calculi types: calcium oxalate $(\mathrm{CaOx})$, cystine, struvite, and uric acid. Types of renal calculi can be 
determined based on physical and chemical features, and several methods of determining the types have been proposed in the literature. Commercial kits measuring the ionic conductivity of samples can be used to determine renal calculi types in a laboratory environment, but while the commercial kits are affordable, the method requires long processing times and cannot differentiate

15 the compounds in a sample [4. The type of renal calculi can also be determined through a material's reaction to heat using the thermogravimetric method. However, the sensitivity of this method is not adequate, and it permanently damages the discarded renal calculi sample [5, 6]. Another method is polarised microscopy, where sample fragments are examined by adding a refractive index 20 liquid. The drawback of this method is the inability to perform component distinction; therefore, this method suffers from low accuracy rates [7. Infrared radiation has also been investigated to determine renal calculi type, but the results of a study were inconclusive due to a similarity to the absorbance range of some stone types [8]. X-ray powder diffraction method is widely used because of the unique diffraction patterns of the different renal calculi types 9, 10. However, this method requires cumbersome sample preparation as well as a trained radiologist. Considering the high cost, difficulty of application, low efficacy, and low sensitivity of the listed methods, it is clear that there is a need for new modalities to classify the stones. One such technique, recently proposed by [11,

30 collects pictures of the renal calculi samples with a standard camera, and a random forest classifier is employed to categorize the renal calculi based on the color and texture of the samples. The obtained accuracies ranged from $63 \%$ to $83 \%$, helping to prove that machine learning algorithms can be effectively utilized for classification of renal calculi, and the accuracy can be increased with data collection. Nevertheless, visual examination may not reveal enough information regarding the molecular structure of renal calculi. This factor may contribute to relatively low accuracy rates because samples with similar appearances may have different molecular structures.

Table 1: Summary of previously reported studies on renal calculi dielectric properties.

\begin{tabular}{llll}
\hline Study & Frequency & Temperature $\left({ }^{\circ} \mathrm{C}\right)$ & Type \\
\hline \hline$[12]$ & $10 \mathrm{kHz}-1.5 \mathrm{MHz}$ & N/A & $\begin{array}{l}\text { Natural stones (solid } \\
\text { and powdered) }\end{array}$ \\
\hline$[13]$ & $1 \mathrm{kHz}-1 \mathrm{MHz}$ & $30-80$ & Single diffusion gel \\
\hline$[14]$ & $1 \mathrm{~Hz}-1 \mathrm{MHz}$ & R.T. & Phantoms \\
\hline$[15]$ & $100 \mathrm{kHz}-1 \mathrm{MHz}$ & $40-110$ & Silica gel \\
\hline$[16]$ & $1 \mathrm{~Hz}-1 \mathrm{MHz}$ & $40-100$ & $\begin{array}{l}\text { Natural stones (pow- } \\
\text { dered) }\end{array}$ \\
\hline$[17]$ & $2.2 \mathrm{GHz}-2.9 \mathrm{GHz}$ & N/A & $\begin{array}{l}\text { Natural stones and } \\
\text { crystals grown }\end{array}$ \\
\hline \hline
\end{tabular}


One other approach that has not been explored in the literature is the employment of dielectric properties for classification of the discarded renal calculi. Dielectric properties are associated with the molecular structure of a material, and it governs the interactions between electromagnetic waves and materials. Thus, the dielectric properties of a medium can be determined by analyzing the electromagnetic wave behavior in that medium. In order to exploit potential 45 microwave diagnostic and therapeutic technologies, researchers have performed extensive microwave dielectric property analysis on different biological tissues and biomaterials [18, 19, 20]. However, microwave dielectric properties of renal calculi have not been fully explored in the literature. Reported studies mostly cover the low frequencies and require machining of the renal calculi. These ${ }_{50}$ studies are performed with the motivation of providing insight into the disease pathogenesis in order to develop new treatment techniques. In [13, the dielectric properties of the grown struvite crystal were studied at low frequencies to investigate the potential for heat dissipation leading to the disintegration of the renal calculi. In [14, an evaluation of the stress effect on renal calculi phantoms 55 was studied in five phantom categories. In [15, the dielectric properties of calcium oxalate grown in silica gel were reported in order to reveal pathogenesis of renal calculi. [16] investigated the growth mechanism of renal calculi by interpreting the dielectric constant via X-ray diffraction (XRD) analysis. In [17, the microwave cavity perturbation technique was used to collect and compare ${ }_{60}$ the dielectric properties of in-vitro and naturally formed stones. However, the reported dielectric properties only cover a narrow band and the study only analyzed a small number of samples. Reported studies on dielectric properties of renal calculi are listed in Table 1, where the listed studies performed below 1.5 $\mathrm{MHz}$ employed parallel plate technique to measure the dielectric properties.

65 This study proposed to employ the open-ended coaxial probe technique to collect dielectric property measurements. In comparison to the techniques previously employed in studies, the open-ended coaxial probe technique requires minimal sample preparation (e.g., machining of the sample is not required) and is able to perform broadband dielectric property measurement. In this work,

70 dielectric properties of samples belonging to three different renal calculi types of calcium oxalate, cystine, and struvite were measured between $500 \mathrm{MHz}$ to $6 \mathrm{GHz}$. One pole Cole-Cole equations, a mathematical expression frequently used for expressing the dielectric property behavior of biological tissues over wide frequency ranges, were fitted to the measurements, and the parameters of 75 the Cole-Cole equations were used as features for the k-nearest neighbors algorithm. The algorithm was then utilized for determining the class of renal calculi samples. The promising results indicated that the technique can be employed for rapid determination of discarded renal calculi types to enable the necessary measures for prevention of the disease.

This paper is organized as follows: dielectric property measurement and the machine learning algorithm are explained in Section 2. The sample preparation and dielectric property measurement setup are given in Section 3 . The dielectric property measurements are provided in Section 5.1, and the $\mathrm{kNN}$ algorithm results are given in Section 5.3. Finally, the conclusions drawn are discussed in 


\section{Background}

The microwave dielectric properties, namely permittivity and conductivity have been widely used to exploit the diagnostic and therapeutic potential of microwaves. This is enabled by the inherent dielectric property discrepancy between healthy and abnormal tissues. Although the dielectric properties of many different biological tissues and biological anomalies have been widely reported in the literature, there are very few reported studies on the microwave dielectric property behavior of renal calculi. This work explored the inherent dielectric property discrepancy between different renal calculi types and exploited this 95 property to classify the discarded samples by utilizing a machine learning algorithm. In this section, we first emphasize the significance of the open-ended coaxial probe dielectric property measurement method and explain the features that were given as inputs to the $\mathrm{kNN}$ algorithm. Both the open-ended coaxial probe technique and the $\mathrm{kNN}$ algorithm are explained in great length in the literature; thus, both topics are discussed only briefly in this work.

\subsection{Open-ended coaxial probe technique}

Dielectric properties of biomaterials have been widely investigated to enable the advancements in microwave diagnostic and therapeutic technologies. Different techniques have been used for measurement of dielectric properties, and the open-ended coaxial probes. Choosing the proper technique depends on the frequency, material properties, and application requirements. For example, the parallel plate technique is suitable for low-frequency measurements and requires heavy machining of the sample. Similarly, the cavity perturbation tech110 nique can only perform narrowband measurements, and the sample needs to be heavily machined. On the other hand, the open-ended coaxial probe technique is simple to operate, does not require machining of the sample, and is able to perform broadband measurements 21. The technique is mostly used for dielectric property measurements of biological materials with high permittivity and loss. Despite all the advantages, the technique is only utilized in laboratory environments, and multiple measurements from one sample are required due to high error and low measurement repeatability rates.

Advantages of the technique make it attractive for using to measure renal calculi dielectric properties. In this study, we aimed to eliminate the heavy machining of the sample, and the disadvantages of the technique were mitigated via the machine learning algorithm. Therefore, this study enables the classification of renal calculi with a single dielectric property measurement. In addition, the discarded renal calculi sample will not be destroyed and can be used for further processing.

125 One important requirement of this technique is to place the aperture of the probe on the sample under test without leaving an air gap. To provide direct 
contact, all renal calculi samples, which tend to have rough surface, were sanded to make their surfaces smoother. The open-ended coaxial probe measurement system used in this study consisted of a vector network analyzer (VNA) commercial software to calculate dielectric properties, and a coaxial probe kit, as shown in Fig. 1

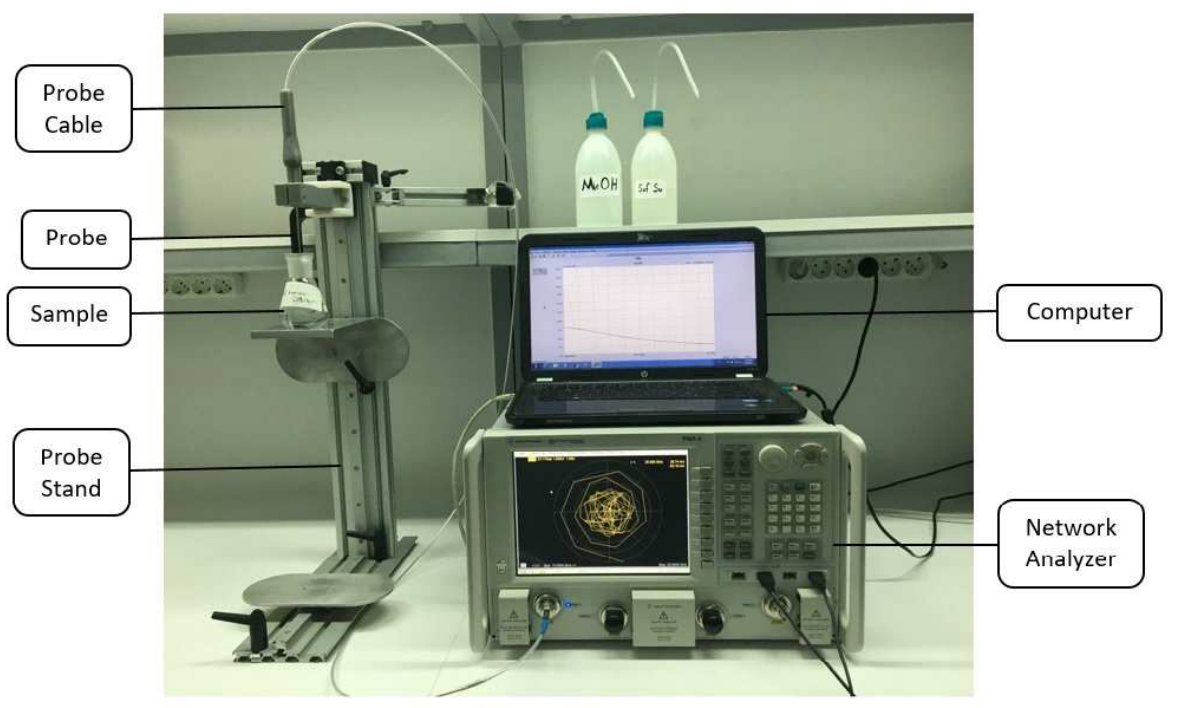

Figure 1: The open-ended coaxial probe set-up for dielectric property measurement.

VNA essentially consists of a signal source, a receiver, and a display. VNA is used for measuring microwave network parameters; that is, scattering parameters (S-parameters). The source transmits the signal to the renal calculi through a coaxial probe, and the receiver measures the signal reflected back to the probe from the sample. After obtaining S-parameters, dielectric constant and dielectric loss values of each stone have been computed over the frequency range of $500 \mathrm{MHz}$ to $6 \mathrm{GHz}$ with $100 \mathrm{MHz}$ intervals by the commercial software.

\subsection{Machine learning algorithm: the k-nearest neighbors (kNN)}

Predictive or descriptive machine learning algorithms have been applied to solve many different problems, including but not limited to data mining, natural language processing, image recognition, and expert systems. Machine learning algorithms are known to be particularly effective when the exact relationship between inputs and outputs of a process are not apparent. Machine learning,

145 a subfield of artificial intelligence, refers to the computer's ability to learn from training data or past experiences and successfully generalize the model to a test set. In machine learning algorithms, data can be processed with supervised or unsupervised learning approaches. Both inputs and their corresponding outputs 
are used in supervised machine learning algorithms, while unsupervised ones uti-

lize only inputs to train the algorithm. Considering this description, the type of machine learning algorithm is determined by input types, the presence of outputs, and desired outputs. For classification of renal calculi, supervised machine learning models such as support vector machine (SVM), artificial neural network (ANN), k-nearest neighbors ( $\mathrm{kNN}$ ) and other supervised learning algo-

155 rithms, can be used since both the inputs and the outputs of the training set are known.

In 22, negligible differences were observed between the SVM and kNN algorithms with data sets that had few or moderate number of features. However, SVM aims to find the best classification function to discriminate the samples of 160 two classes in the training set. Since it is a binary classifier, it needs to be modified for a multi-class problem [23. In the study conducted by [24, cumulative error performances of machine learning algorithms were analyzed with respect to sample size. According to the study, the kNN algorithm showed better performance for data sets that were closest to our sample size. As a consequence, the $\mathrm{kNN}$ algorithm was preferred for the present study in order to decrease the complexity and improve the performance of classification.

The kNN algorithm works by categorizing data via correlating inputs to similar outputs. In a sense, as the algorithm confronts unknown data, it investigates similar instances from the training set. Two design parameters - the 170 number of nearest neighbors, $\mathrm{k}$, and the distance between data points - were adjusted while developing the $\mathrm{kNN}$ model. The distance could be calculated with Euclidean distance, Manhattan distance, or Minkowski distance relations. To train the model, data in the training set were positioned on a coordinate system that is compatible with the data dimensions. During the testing stage,

175 unknown data were placed into same coordinate system to specify the kNN. The class of unknown data, then, was estimated from the majority classes of neighbors.

\section{Experiments}

A detailed work on the dielectric properties of three renal calculi categories, calcium oxalate, cystine, and struvite, was carried out for classification. Additionally, another study was performed by the authors on the dielectric properties of renal calculi with a limited number of samples covering the frequency range of $0.5 \mathrm{GHz}$ to $18 \mathrm{GHz}[25$. Complex permittivity data composed of dielectric constant $\left(\epsilon^{\prime}\right)$ and dielectric loss factor $\epsilon^{\prime \prime}$ were obtained via the open-ended coaxial probe technique, which is widely used to measure broadband dielectric properties of biomaterials in a laboratory environment. The properties of the renal calculi samples, the experimental setup, and the classification procedures used are detailed in the following sections.

\subsection{Samples}

${ }_{190}$ A total of 105 naturally occurring renal calculi samples were obtained from the Department of Urology, Cerrahpasa Medical School. The renal calculi sam- 
ples were gathered from 40 patients by utilizing various treatment methods including percutaneous nephrolithotomy (PCNL). The samples obtained were sorted into two groups, where the first group included a total of 49 samples with 21 calcium oxalate, 7 cystine, and 11 struvite renal calculi samples. The second group included a total of 66 samples with 14 calcium oxalate, 28 cystine, and 24 struvite renal calculi samples. While the obtained number of renal calculi samples was greater than 105, some of the samples were eliminated in both measurement periods due to their small dimensions. The radius of measured samples used ranged from $2.5 \mathrm{~mm}$ to $10 \mathrm{~mm}$. Pictures from the obtained renal calculi samples are shown in Fig. 2. The sample preparation was minimally laborious compared to other methodologies. Since the aperture of the probe has to be fully in contact with the material being tested, the surfaces of the renal calculi samples were sanded lightly to obtain a flat surface.

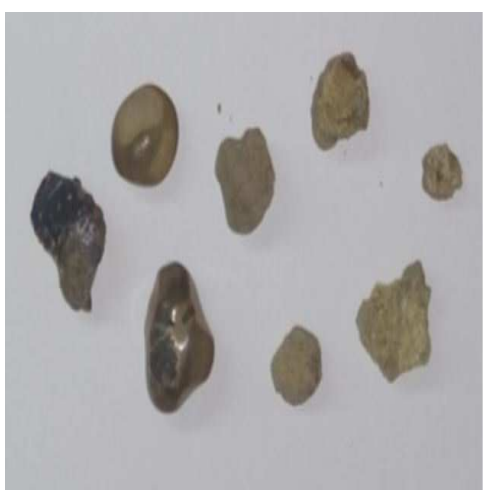

(a)

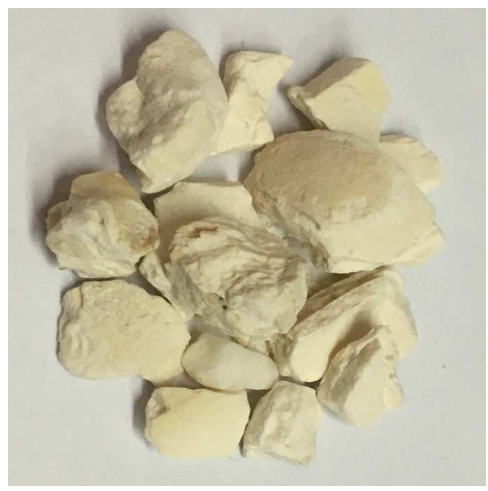

(c)

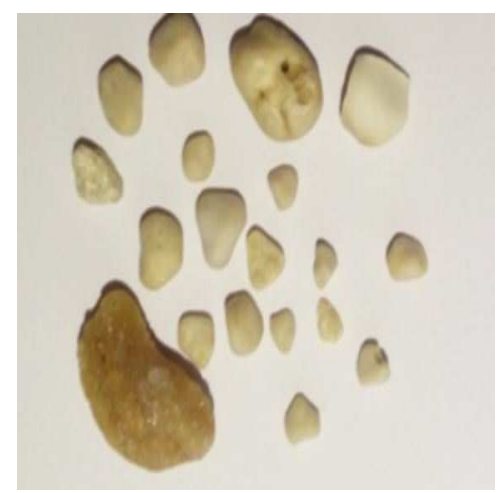

(b)

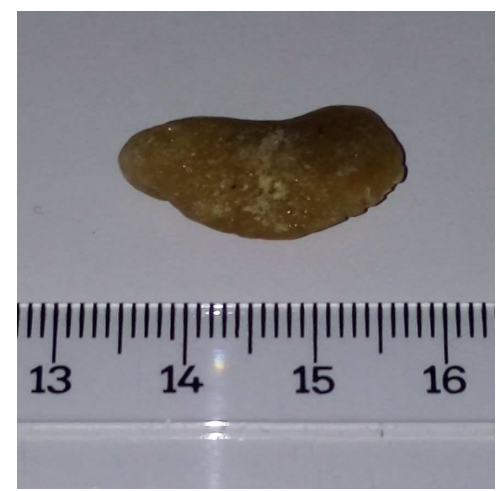

(d)

Figure 2: Some of the renal calculi samples utilized for dielectric property measurements, (a) a portion of the calcium oxalate samples, (b) a portion of the cystine samples, (c) a portion of the struvite samples, (d) a cystine sample. 


\subsection{Experimental setup}

The dielectric properties of the renal calculi samples were measured using a slim-form open-ended coaxial probe with an aperture size of $2.2 \mathrm{~mm}$. The S-parameters were measured with an Agilent N5245A PNA-X Microwave Network Analyzer (shown in Fig. 1). The PNA-X was connected to the probe with a $50 \Omega$ RF-cable. Both the VNA and the open-ended coaxial probe have characteristic impedance of $50 \Omega$ as well, which ensures impedance matching. The impedance of the open-ended coaxial probe was determined by the diameters of the inner and outer conductors and the relative permittivity of the dielectric material sandwiched between them. The probe utilized in this work had an outer conductor diameter of $2.2 \mathrm{~mm}$ and an inner conductor diameter of 0.6 $\mathrm{mm}$, and the material between the concentric conductors was Teflon. The VNA was also connected via local area network (LAN) to a notebook computer, where a commercially available Agilent $85070 \mathrm{E}$ software was used for converting the scattering parameters to material dielectric properties.

\subsection{Measurement procedure}

Before starting the measurement process, the slim-form dielectric probe was calibrated by implementing the standard open, short, and deionizedwater calibration procedure. After performing the calibration, complex permittivity measurements of pure methanol were collected to validate the calibration. Then the probe aperture was pressed against the flat surface of the renal calculi sample, ensuring that the probe tip was fully in contact with the sample. At least five measurements were obtained from different points on each sample surface whenever appropriate. The measurements were taken between $500 \mathrm{MHz}$ and $6 \mathrm{GHz}$, with $100 \mathrm{MHz}$ intervals.

\section{Methods}

Measured dielectric properties are expressed with Cole-Cole equation. First, the median of measurements collected from each sample was calculated. Next, the Cole-Cole parameters were fitted to the median dielectric property data.

\subsection{Measured wideband dielectric property analysis}

In the literature, dielectric properties of biological materials are widely represented with a Cole-Cole model formulated as follows [26]:

$$
\epsilon^{\prime}-i \epsilon^{\prime \prime}=\epsilon_{\infty}+\frac{\epsilon_{s}-\epsilon_{\infty}}{(1+i w \tau)^{(1-\alpha)}}+\frac{\sigma}{i w \epsilon_{0}}
$$

where $\epsilon^{\prime}$ is the dielectric constant, $\epsilon^{\prime \prime}$ is the dielectric loss factor, $\epsilon_{\infty}$ and $\epsilon_{s}$ are dielectric constants measured at higher and lower frequencies, respectively, $\mathrm{w}$ is angular frequency, $\tau$ is relaxation time, $\alpha$ is the distribution of relaxation time, and $\sigma$ is the ionic conductivity of the sample. The terms, $\epsilon_{\infty}, \epsilon_{s}, \tau, \alpha$, and $\sigma$, are called Cole-Cole parameters. 
In this study, by substituting measured $\epsilon^{\prime}$ and $\epsilon^{\prime \prime}$ values of renal calculi samples in a $500 \mathrm{MHz}$ to $6 \mathrm{GHz}$ frequency range into (1), an equation with five unknown Cole-Cole parameters was obtained at each measurement frequency point for a single stone. In order to find the Cole-Cole parameters, the equation set of each sample was solved by the generalized Newton-Raphson (GNR) method, which is a numerical method making use of the partial derivatives of the equation. The error in GNR is defined as the Euclidean distance of the calculated data to the measured data given in (2):

$$
\text { error }=\frac{1}{N} \sum_{i=1}^{N}\left[\left(\frac{\epsilon_{w_{i}}^{\prime}-\hat{\epsilon}_{w_{i}}^{\prime}}{\operatorname{median}\left(\epsilon_{w_{i}}^{\prime}\right)}\right)^{2}-\left(\frac{\epsilon_{w_{i}}^{\prime \prime}-\hat{\epsilon}_{w_{i}}^{\prime \prime}}{\operatorname{median}\left(\epsilon_{w_{i}}^{\prime \prime}\right)}\right)^{2}\right]
$$

where $\epsilon_{w_{i}}^{\prime}$ and $\epsilon_{w_{i}}^{\prime \prime}$ are the measured dielectric properties obtained with the open-ended coaxial probe technique and $\hat{\epsilon}_{w_{i}}^{\prime}$ and $\hat{\epsilon}_{w_{i}}^{\prime \prime}$ are calculated dielectric properties. In this work, these dielectric properties were calculated with the Cole-Cole equation to evaluate the performance of the suggested Cole-Cole parameters. Last, $\mathrm{N}$ is the number of points used within the frequency range of $0.5 \mathrm{GHz}$ to $6 \mathrm{GHz}$ 27. N was set at 56 in this study, which is the number of points at the measured frequency of interest. The calculated Cole-Cole parameters were saved to use as inputs for the classification algorithm. The iterative process stops when the algorithm finds the first Euclidean distance below a determined threshold and picks the Cole-Cole parameters that result

260 in a minimum Euclidean distance. Note that the threshold picked in this study was 0.05 .

\subsection{Classification of renal calculi samples}

Renal calculi samples were categorized using the kNN algorithm, where the calculated Cole-Cole parameters were given as inputs to the classification algorithm. In the first stage, input vectors were normalized in the range of $(-1,1)$ since normalization allows faster convergence during training. After normalization, the data were divided into training and testing sets by applying 10 fold cross-validation. In this method, the data set was split into 10 equal folds. During each round, the data in nine folds were used for training, and the remaining fold was used for testing. A fair test of validation for limited sample size can be obtained with 10 fold cross-validation by using features for both training and testing.

In our renal calculi classifier, $\mathrm{k}$ was set to 10 because it is a common practice to set the k parameter equal to the square root of the number of training samples 275 28. The distance parameter chosen was Euclidean distance. By utilizing these parameters, the $\mathrm{kNN}$ model was then trained and tested. The performance of the kNN model was calculated with a two-by-two confusion matrix consisting of true positive (tp), false positive (fp), false negative ( $\mathrm{fn})$, and true negative $(\mathrm{tn})$ counts in the classification. In this study, accuracy, sensitivity, specificity, precision, recall, and F1 score performance measures were calculated from confusion matrices and used to evaluate the model. 


\section{Results}

Section 5.1 reports the dielectric measurement results for the renal calculi samples, the Cole-Cole fitting results for the dielectric properties are given in Section 5.2 , and Section 5.3 describes the renal calculi classification results using $\mathrm{kNN}$.

\subsection{Dielectric measurement results of renal calculi samples}

The median dielectric properties of the renal calculi samples that were calculated from measurements collected with the open-ended coaxial probe are represented with variability bars in Fig 3(a) and Fig. 3(b). To better emphasize the dielectric property discrepancy between stone types, the median permittivity and conductivity values of calcium oxalate, cystine, and struvite samples are given in Table 2 at six different frequency points. Medians of the measured relative permittivity of calcium oxalate, cystine, and struvite between

$2951 \mathrm{GHz}$ and $6 \mathrm{GHz}$ were ranges of, respectively, 2.28 to $2.35,2.17$ to 2.63 and 3.16 to 3.38 . The median conductivity of calcium oxalate, cystine, and struvite between $0.5 \mathrm{GHz}$ and $6 \mathrm{GHz}$ were ranges of, respectively, $0.45 \times 10-2\left(\mathrm{~S} \mathrm{~m}^{-1}\right)$ to 1.6x10-2 (S $\left.m^{-1}\right), 0.19 \times 10-1\left(\mathrm{~S} \mathrm{~m}^{-1}\right)$ to $1.5 \times 10-1\left(\mathrm{~S} \mathrm{~m}^{-1}\right)$, and 2.0x10-2 $\left(\mathrm{S} \mathrm{m}^{-1}\right)$ to $3.5 \times 10-2\left(\mathrm{~S} \mathrm{~m}^{-1}\right)$. The permittivity discrepancy between renal calculi types 300 tends to decrease with increasing frequency, whereas the conductivity discrepancy increases with increasing frequency. Cystine had the lowest and struvite had the highest relative permittivity at microwave frequencies as seen in Fig. 3(a). Although the conductivity parameter was very low for all stone types, the conductivity of struvite was relatively higher than the other two types.

Table 2: Median of dielectric property measurements for calcium oxalate, cystine, and struvite.

\begin{tabular}{ccccccc}
\hline \hline \multirow{2}{*}{$\begin{array}{c}\text { Frequency } \\
(\text { GHz })\end{array}$} & \multicolumn{2}{c}{ Calcium Oxalate } & \multicolumn{2}{c}{ Cystine } & \multicolumn{2}{c}{ Struvite } \\
\cline { 2 - 7 }$\epsilon^{\prime}$ & $\sigma\left(\mathrm{S} \mathrm{m}^{-1}\right)$ & $\epsilon^{\prime}$ & $\sigma\left(\mathrm{S} \mathrm{m}^{-1}\right)$ & $\epsilon^{\prime}$ & $\sigma\left(\mathrm{S} \mathrm{m}^{-1}\right)$ \\
\hline 1.0 & 2.3525 & 0.0045 & 2.6335 & 0.0190 & 3.3131 & 0.0199 \\
2.0 & 2.3067 & 0.0079 & 2.5113 & 0.0481 & 3.3779 & 0.0269 \\
3.0 & 2.3271 & 0.0091 & 2.3991 & 0.0760 & 3.2421 & 0.0265 \\
4.0 & 2.2392 & 0.0113 & 2.3241 & 0.1342 & 3.1628 & 0.0275 \\
5.0 & 2.2771 & 0.0150 & 2.3675 & 0.1498 & 3.3729 & 0.0303 \\
6.0 & 2.3226 & 0.0161 & 2.1708 & 0.1491 & 3.2165 & 0.0349 \\
\hline
\end{tabular}

\subsection{Cole-Cole fitting results for renal calculi dielectric properties}

The Cole-Cole parameters were fitted to the measured relative permittivity and conductivity of each stone sample by utilizing GNR method. Two examples of the Cole-Cole fitting to median measurements of each type are shown in Fig. 4(a) and Fig. 4(b). A good agreement was achieved between the median of 310 the measurement data and the Cole-Cole fitting. A comparison of the fitted 


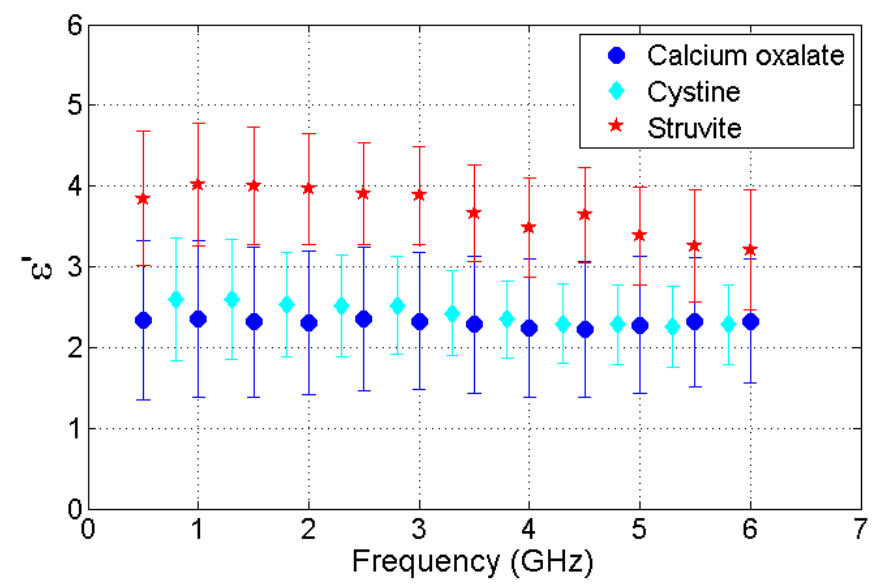

(a)

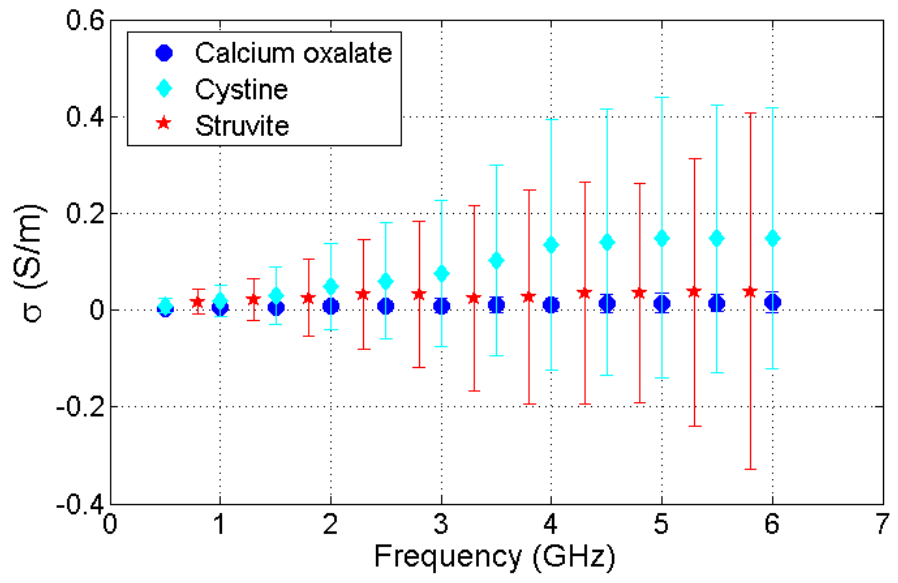

(b)

Figure 3: Median dielectric property measurements of renal calculi samples with variability bars, (a) median relative permittivity measurement, (b) median conductivity measurement.

Cole-Cole parameters is given in Table 3. The error parameter of Euclidean distance was lower than the threshold value. One can classify the renal calculi just by looking into the Cole-Cole parameters or median measurements. However, the measurement system suffered from low measurement accuracy and low repeatability rates. Therefore, machine learning algorithm was implemented to compensate for the errors stemming from the measurement methodology and other systematic errors. 


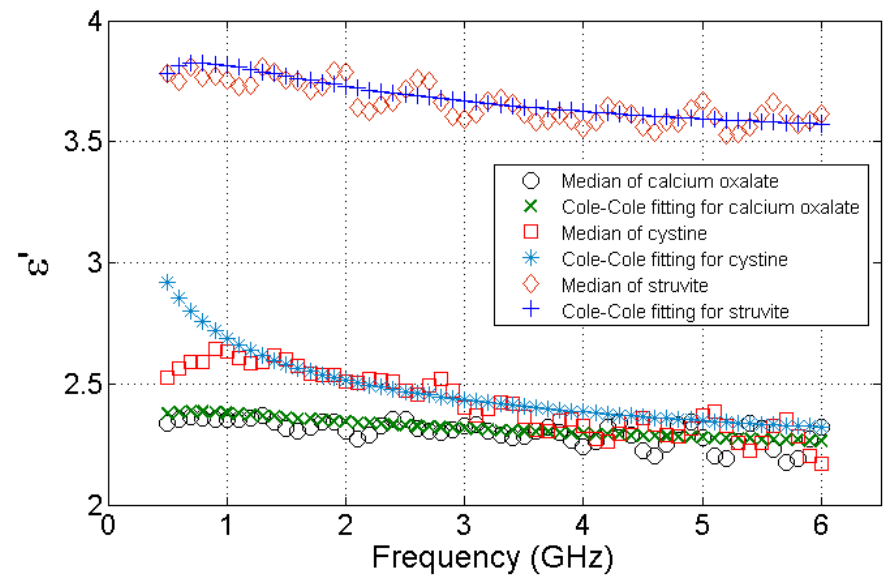

(a)

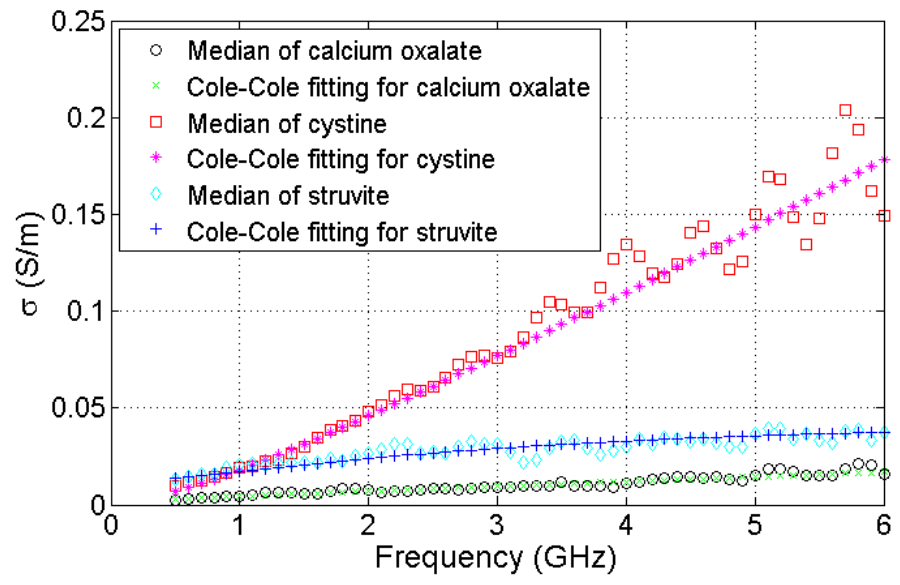

(b)

Figure 4: Comparison of calculated median values with ColeCole fittings, (a) relative permittivity comparisons for calcium oxalate, cystine, and struvite samples, (b) conductivity comparisons for calcium oxalate, cystine, and struvite samples.

\subsection{Renal calculi classification results with $k N N$}

We have tested our method on a data set collected from 35 calcium oxalate, 35 cystine, and 35 struvite renal calculi samples. Training and testing data were selected by applying 10 fold cross-validation to avoid over-fitting and to achieve unbiased classification results. Calculated performance measures are demonstrated in Fig. 5 .

As seen from Fig. 5, all performance measures of this classifier reached to $100 \%$ in at least one fold. Among 10 folds, the minimum obtained values of ac- 
Table 3: A sample of Cole-Cole parameters fitted to the median of each stone type.

\begin{tabular}{ccccccc}
\hline \hline Cole-Cole parameters & $\epsilon_{\infty}$ & $\epsilon_{s}$ & $\tau$ & $\alpha$ & $\sigma\left(\mathrm{S} \mathrm{m}^{-1}\right)$ & error \\
\hline CaOx & 1.96 & 12.84 & $1.41 \mathrm{e}-06$ & 0.73 & $8.11 \mathrm{e}-05$ & 0.0151 \\
Cystine & 1.93 & 16.78 & $6.04 \mathrm{e}-07$ & 0.62 & 0.0071 & 0.0111 \\
Struvite & 3.50 & 14.66 & $1.80 \mathrm{e}-07$ & 0.39 & 0.0365 & 0.0099 \\
\hline
\end{tabular}

curacy, sensitivity, specificity, precision, recall, and F1 score were, respectively, $90.00 \%, 80.00 \%, 85.71 \%, 75.00 \%, 87.50 \%$, and $88.89 \%$. These results are quite promising for categorizing the renal calculi based on their dielectric properties. By further testing the model with a larger data set, a new renal calculi analysis tool could emerge that can be utilized in hospitals, research centers, and laboratories.

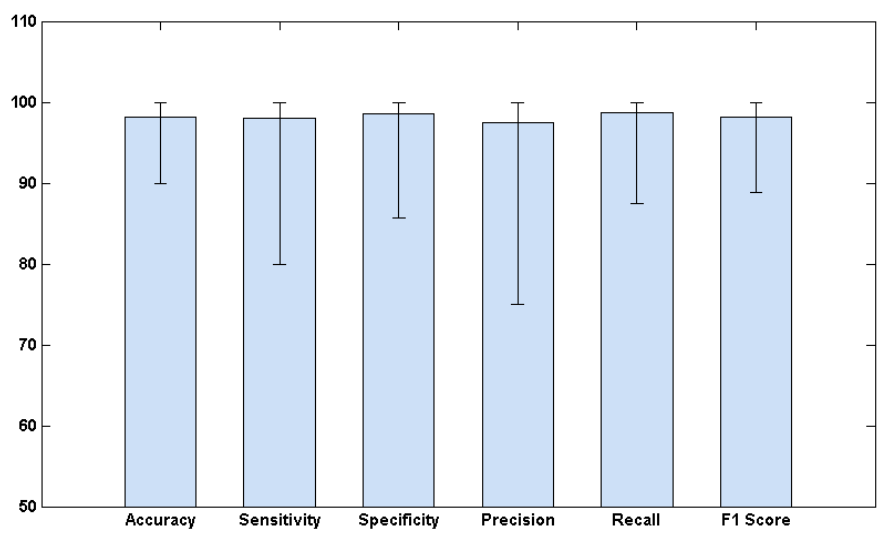

Figure 5: Performance measures of the $\mathrm{kNN}$ for classification of all renal calculi samples. Error bars show the maximum and minimum values of performance measures among the 10 folds used in testing.

\section{Discussion}

The prevalence of renal calculi and the types depend on the geographic, climatic, and dietary conditions as well as the race, sex, and age of the patient.

335 Therefore, the availability of these samples depends on the patients visiting a clinic, the location of the clinics, and also the severity of the disease (e.g., the renal calculi can be disintegrated in vivo by response to a nonsurgical treatment such as oral medications and extracorporeal shockwave lithotripsy (ESWL) allowing it to be passed naturally). Uric acid is a relatively rarely diagnosed renal 

lence varies geographically, being $2.1 \%$ in Texas, USA, and $15.8 \%$ in Okinawa, Japan 29. More importantly, uric acid is known to be soluble in high pH urea. Since it is known to be responsive to nonsurgical treatments, for patients with uric acid renal calculi, oral chemolysis is generally prescribed [30, 31]. Most urolithasis patients with uric acid respond well to oral medications. In some cases, this treatment is accompanied with ESWL and PCNL, and PNCL is preferred when the uric acid sample is larger than $2 \mathrm{~cm} \mathrm{32}$. Due to the high solubility of uric acid renal calculi, the uric acid samples were not available at the hospital. Hence, uric acid was not included in this work.

To compare the performance of the $\mathrm{kNN}$ for three renal calculi types with other machine learning algorithms, we applied the ANN method to the problem of classification of renal calculi. The ANN algorithm mimics the neural structure of the brain and has been utilized in other studies for different tasks, including but not limited to autonomous driving and medical diagnostics. Briefly, the ficial neuron inputs mimic the dendrites, node represents the soma, and the weighted output represents the axon in a biological neuron. The nodes sum the weighted inputs and pass them from an activation function that can be a linear, step, or sigmoid function. In this study, a tangent sigmoid function was used

360 as an activation function. Then the output was taken and passed to another layer of concatenated neurons. The ANN algorithm works by optimizing the weights of the inputs and outputs via gradient descent backpropagation. The number of layers and inputs depend on the neural net, and for this work, it was determined via trial and error. In this work, we employed one hidden layer with eters along with bias, and the outputs of the network were the three classes of calcium oxalate, cystine, and struvite. The inputs were normalized in the range of $(-1,1)$ since the normalization enabled fast convergence. Finally, the chosen learning rate was between 0 to 1 , adjusted along with the weights through back 370 propagation during training. The training and testing were then performed, and the results of the ANN algorithm, as well as the $\mathrm{kNN}$ results, are given in Table 4 .

As seen in Table 4, kNN outperformed the ANN, especially when the F1 score is considered. The performance of the ANN could be further optimized by adopting different activation functions or even by adding layers to the neural network. Ultimately, we expect that the performance of both algorithms would improve with a significant increase in sample size. One should also consider that the computational cost of $\mathrm{kNN}$ is higher for more data.

When we analyzed the dielectric property data, the median relative permittivity of struvite was approximately 1 unit higher than cystine and calcium oxalate for all frequencies between $0.5 \mathrm{GHz}$ and $6 \mathrm{GHz}$, as shown in Fig. 4(a). Therefore, we can state that when the relative permittivity was considered, struvite separated quite well from the other two classes. Similarly, it can be seen from Fig. 4(b) that the conductivity of the cystine seperated well from the other 
Table 4: A comparison of performance measures of ANN and kNN algorithms employed for renal calculi classification.

\begin{tabular}{ccc}
\hline \hline Performance Measures & ANN & kNN \\
\hline Accuracy (\%) & 98.1 & 98.2 \\
Sensitivity (\%) & 97.1 & 98.0 \\
Specificity (\%) & 98.6 & 98.6 \\
Precision (\%) & 97.2 & 97.5 \\
Recall (\%) & 98.6 & 98.8 \\
F1 Score (\%) & 96.0 & 98.1 \\
\hline
\end{tabular}

permittivity and conductivity could be two parameters that may help seperate the classes.

Finally, to the best of authors' knowledge, classification based on the dielectric property measurement has not been previously explored in the literature, except in 27, where a binary classification algorithm SVM is applied to the collected dielectric property data, and malignant hepatic tissues were classified with a $99.2 \% \mathrm{~F} 1$ score. As the dielectric property contrast grows, the performance of the machine learning algorithms slightly increases. However, we can clearly state that despite the relatively low dielectric property contrast in this 395 work (relative permittivity discrepancy between the malignant and healthy hepatic tissues in [27] was 5 to 10 units), the machine learning algorithms were very effective for classifying discarded renal calculi.

\section{Conclusion}

A microwave dielectric property based $\mathrm{kNN}$ renal calculi classification method was presented in this work. Dielectric properties of three different renal calculi types were measured with the slim-form open-ended coaxial probe technique between $500 \mathrm{MHz}$ and $6 \mathrm{GHz}$, with $100 \mathrm{MHz}$ frequency steps. The medians of the dielectric properties were calculated, and an inherent dielectric property discrepancy was observed in the different renal calculi types. The Cole-Cole parameters were then fitted to measurement data, which aided in the representation of dielectric property measurement data with only five parameters. The $\mathrm{kNN}$ algorithm was then employed for classification of the renal calculi, and the Cole-Cole parameters were used for training and testing the algorithm. The benefits of the proposed method include rapid measurement, minimal sample 410 preparation requirements, and an automated decision making mechanism that can eliminate personnel costs, decrease diagnosis time, and decrease equipment costs. It should also be noted that the system itself is simple and requires very little output power. In addition, unlike X-rays, no special chamber is required to confine the low-power microwaves. 
A good performance (98.17\% accuracy) was achieved for renal calculi classification with a $\mathrm{kNN}$ model based on the Cole-Cole parameters. However, there could still be some error sources that may decrease the method's performance, which can be categorized as measurement errors, data analysis errors, or the diversity of samples. Measurement errors can be due to an air gap between

${ }_{420}$ the samples and aperture of the probe. Data analysis errors can stem from the errors that emerge due to representation of the data with few parameters. For example, Cole-Cole parameters that can represent a curve are not necessarily unique, and different combinations can satisfy the requirements. Finally including a more diverse data set containing more than 105 renal calculi samples could improve performance of the machine learning algorithm.

\section{Acknowledgements}

This work was partially supported by the European Unions Horizon 2020 research and innovation program under the Marie Sklodowska-Curie grant agreement No. 750346.

\section{References}

[1] V. Romero, H. Akpinar, D. .-. e. Assimos, Kidney stones: a global picture of prevalence, incidence, and associated risk factors, Reviews in urology $12(2-3)(2 / 3)(2010)$ e86-e96.

[2] D. A. Schulsinger, Kidney Stone Disease, Springer International Publishing, 2015.

[3] K. Andreassen, A. Paulsen, P. Olsen, J. Aabeck, P. Osther, Classification of urolithiasis in denmark: a national survey, European Urology 2 (1) (2007) 126.

[4] I. A. Hashim, T. H. Zawawi, Wet vs. dry chemical analysis of renal stones, Irish Journal of Medical Science 168 (1999) 114.

[5] G. Rose, C. Woodfine, The thermogravimetric analysis of renal stones (in clinical practice)., British Journal of Urology 48 (6) (1976) 403-412.

[6] H. Lee, D. Leong, C. Heng, Characterization of kidney stones using thermogravimetric analysis with electron dispersive spectroscopy., Urological Research 40 (3) (2012) 197-204.

[7] D. Douglas, D. Tonks, The qualitative analysis of renal calculi with the polarising microscope, Clinical Biochemistry 12 (5) (1979) 182-183.

[8] G. Kravdal, D. Helgo, M. Moe, Infrared spectroscopy is the gold standard for kidney stone analysis, Tidsskr Nor Laegeforen 135 (4) (2015) 313-314. 
[9] V. Uvarov, I. Popov, N. Shapur, T. Abdin, O. Gofrit, D. Pode, M. Duvdevani, X-ray diffraction and sem study of kidney stones in israel: quantitative analysis, crystallite size determination, and statistical characterization., Environ Geochem Health 33 (6) (2011) 613-622.

[10] J. Joost, R. Tessadri, Combined analysis of kidney stones by x-ray diffraction and electron microprobe, European Urology 9 (5) (1983) 305-311.

[11] J. Serrat, F. Lumbreras, F. Blanco, M. Valiente, M. Lpez-Mesas, mystone: A system for automatic kidney stone classification, Expert Systems With Applications 89 (2017) 41-51.

[12] V. Singh, J. Lafaut, M. Weversa, C. Vincent, L. Baed, Stress-generated potential in renal calculi and lithotripsy, Proceedings of The First Joint BMES/EMBS conference, 1999.

[13] R. Rajan, N. Raj, S. Madeswaran, D. Babu, Dielectric studies on struvite urinary crystals, a gateway to the new treatment modality for urolithiasis, Spectrochimica Acta Part A: Molecular and Biomolecular Spectroscopy 148 (2015) 266-270.

[14] I. K. Kumarpranawa, D. Saranya, A. Raj, Preparation and characterization of kidney stone phantoms with compression strength and dielectric property studies, International Journal of ChemTech Research 8 (5) (2015) 153-157.

[15] M. Abdulsalim, The characteristics, dielectric properties and surface morphology of calcium oxalate monohydrate single crystals grown in silica gel, Journal of Chemical, Biological and Physical Sciences 2 (2) (2012) 962-967.

[16] D. Sinha, K. Anwar, K. Kumari, S. Jaishwal, S. Madeshwaran, S. Keshari, D. Babu, R. Vidya, A. Nambiraj N, Studies on the dielectric properties of natural urinary stones, Advanced Materials Research 584 (2012) 484-488.

[17] I. Paul, G. Varghese, M. A. Ittyachen, K. T. Mathew, A. Lonappan, J. Jacob, S. Kumar, Dielectric properties of urinary stones at microwave frequencies, MICROWAVE AND OPTICAL TECHNOLOGY LETTERS 35 (4) (2002) 297-299.

[18] M. Lazebnik, M. C. Converse, J. H. Booske, S. C. Hagness, Ultrawideband temperature-dependent dielectric properties of animal liver tissue in the microwave frequency range, Physics in medicine and biology 51 (7) (2006) 1941.

[19] M. Lazebnik, D. Popovic, L. McCartney, C. B. Watkins, M. J. Lindstrom, J. Harter, S. Sewall, T. Ogilvie, A. Magliocco, T. M. Breslin, et al., A largescale study of the ultrawideband microwave dielectric properties of normal, benign and malignant breast tissues obtained from cancer surgeries, Physics in Medicine and Biology 52 (20) (2007) 6093. 
[20] T. Yilmaz, R. Foster, Y. Hao, Broadband tissue mimicking phantoms and a patch resonator for evaluating noninvasive monitoring of blood glucose levels, IEEE Transactions on Antennas and Propagation 62 (6) (2014) 30643075 .

[21] Keysight technologies, "basics of measuring the dielectric properties of materials ", accessed on 13/03/2018. 5989-2589EN.pdf

[22] H. Raeisi Shahraki, S. Pourahmad, N. Zare, Important neighbors: A novel approach to binary classification in high dimensional data, BioMed research international 2017.

[23] N. H. Ruparel, N. M. Shahane, D. P. Bhamare, Learning from small data set to build classification model: A survey, in: Proc. IJCA Int. Conf. Recent Trends Eng. Technol.(ICRTET), 2013, pp. 23-26.

[24] B. S. Clarke, J. L. Clarke, Predictive Statistics: Analysis and Inference Beyond Models, Vol. 46, Cambridge University Press, 2018.

[25] T. Yilmaz, B. Sacli, G. Cansiz, S. Joof, C. Aydinalp, M. Cayoren, B. Onal, I. Akduman, Microwave dielectric spectroscopy of renal calculi: A large scale study on dielectric properties from $500 \mathrm{mhz}$ to $18 \mathrm{ghz}$, unpublished (2019).

[26] S. Gabriel, R. Lau, C. Gabriel, The dielectric properties of biological tissues: Iii. parametric models for the dielectric spectrum of tissues, Physics in Medicine \& Biology 41 (1996) 22712293.

[27] T. Yilmaz, M. A. Kl, M. Erdoan, M. ayren, D. Tunaolu, I. Kurtolu, Y. Yaslan, H. ayren, A. E. Arkan, S. Teksz, G. Cancan, N. Kepil, S. Erdamar, M. zcan, I. Akduman, T. Kalkan, Machine learning aided diagnosis of hepatic malignancies through in vivo dielectric measurements with microwaves, Physics in Medicine \& Biology 61 (13) (2016) 50895102.

[28] B. Lantz, Machine learning with R, Packt Publishing Ltd, 2015.

[29] R. Z. Hossain, Y. Ogawa, S. Hokama, M. Morozumi, T. Hatano, Urolithiasis in okinawa, japan: a relatively high prevalence of uric acid stones, International Journal of Urology 10 (8) (2003) 411-415.

[30] M. Honda, K. Yamamoto, C. Momohara, K. Komori, T. Takada, H. Fujioka, Oral chemolysis of uric acid stones, Hinyokika kiyo. Acta urologica Japonica 49 (6) (2003) 307-310.

[31] N. O. Bernardo, A. D. Smith, Chemolysis of urinary calculi, Urologic Clinics of North America 27 (2) (2000) 355-365.

525 [32] A. Abou-Elela, Epidemiology, pathophysiology, and management of uric acid urolithiasis: A narrative review, Journal of advanced research 8 (5) (2017) 513-527. 